\title{
Double blind, randomised controlled clinical trial of hypo-osmolar oral rehydration salt solution in dehydrating acute diarrhoea in severely malnourished (marasmic) children
}

\author{
P Dutta, U Mitra, B Manna, S K Niyogi, K Roy, C Mondal, S K Bhattacharya
}

\begin{abstract}
Aims-To compare the clinical efficacy of hypo-osmolar oral rehydration salt (ORS) solution (224 mmol/1) and standard ORS solution (311 mmol/1) in severely malnourished (marasmic) children having less than $60 \%$ Harvard standard weight for age with dehydrating acute watery diarrhoea.

Methods-In a double blind, randomised, controlled trial, 64 children aged 6-48 months were randomly assigned standard $(\mathbf{n}=32)$ or hypo-osmolar ORS $(\mathbf{n}=32)$. Results-Stool output (52.3 $v 96.6 \mathrm{~g} / \mathrm{kg} /$ day), duration of diarrhoea (41.5 $v 66.4$ hours), intake of ORS (111.5 $v 168.9 \mathrm{ml} / \mathrm{kg} /$ day), and fluid intake (214.6 $v 278.3 \mathrm{ml} / \mathrm{kg}$ / day) were significantly less in the hypoosmolar group than in the standard ORS group. Percentage of weight gain on recovery in the hypo-osmolar group was also significantly less $(4.3 v 5.4 \%$ of admission weight) than in the standard ORS group. A total of $29(91 \%)$ children in the standard ORS group and $32(100 \%)$ children in the hypo-osmolar group recovered within five days of initiation of therapy. Mean serum sodium and potassium concentrations on recovery were within the normal range in both groups. Conclusion-Our findings suggest that hypo-osmolar ORS has beneficial effects on the clinical course of dehydrating acute watery diarrhoea in severely malnourished (marasmic) children. Furthermore, children did not become hyponatraemic after receiving hypo-osmolar ORS. (Arch Dis Child 2001;84:237-240)
\end{abstract}

Keywords: diarrhoea; hypo-osmolar; oral rehydration salts; malnourished; marasmic

Childhood malnutrition and diarrhoea are common in developing countries and are responsible for a high proportion of deaths in children. ${ }^{1-3}$ A single standard formula of oral rehydration salts (ORS) recommended by the World Health Organisation and United Nations Children's Fund (WHO/UNICEF) is considered a safe and effective therapy for most of the children with dehydrating acute diarrhoea. ${ }^{4}$ Although several studies support the use of this standard ORS for the treatment of acute diarrhoea in severely malnourished children, ${ }^{56}$ there are still unresolved issues regarding the optimal salt content of ORS, especially in severely malnourished children. Paediatricians fear that this standard ORS (which contains $90 \mathrm{mmol}$ of sodium) may produce hypernatraemia and over hydration in severely malnourished (marasmic) children, because they excrete less salts and water as a result of changes in renal mechanisms including reduced glomerular filtration and low tubular capacity to concentrate urine. ${ }^{7-9}$ Malnourished children thus run a high risk of accumulating fluid and electrolytes in the body.

Recently, several clinical studies documented that hypo-osmolar solution, containing low sodium and glucose, was better than standard ORS for the treatment of dehydrating acute diarrhoea in children. ${ }^{10-14}$ The low osmolality promoted intestinal absorption of sodium and water. Gastric emptying of hypo-osmolar ORS was enhanced. Furthermore, more complete absorption of glucose reduced the risk of osmotic diarrhoea compared to standard ORS. However, there is no published evidence of the efficacy of hypo-osmolar ORS in severely malnourished children. We therefore performed a double blind, randomised, clinical trial to compare the efficacy of standard and hypo-osmolar ORS in marasmic children suffering from dehydrating acute diarrhoea.

\section{Patients and methods}

The study was carried out at the Dr BC Roy Memorial Hospital for Children, Calcutta, India between July 1997 and August 1999. Male children less than $60 \%$ Harvard standard weight for age without oedema (for ease of collection of stool and urine separately), aged between 6 and 48 months who were marasmic were included in the study if they had a history of acute watery diarrhoea (three or more loose watery stools per day) for 72 hours or less and clinical signs and symptoms of "some" dehydration (for example, thirst or eagerness to drink, sunken eyes, dry mouth and tongue, and loss of skin elasticity). ${ }^{4}$

However, children with the following features were not included in the study: (1) a history of another episode of diarrhoea one month prior to the onset of present illness; (2) had received antibiotics or oral rehydration therapy during this episode of diarrhoea; (3) obvious parenteral infection-septicaemia, meningitis, pneumonia, or urinary tract infection; (4) require special medical care (life support system, blood transfusion, or total parenteral nutrition); (5) exclusively breast fed; (6) obvious signs of kwashiorkor. 
Table 1 Characteristics of patients on admission

\begin{tabular}{lll}
\hline Characteristics & $\begin{array}{l}\text { Standard ORS } \\
(n=32)\end{array}$ & $\begin{array}{l}\text { Hypo-osmolar ORS } \\
(n=32)\end{array}$ \\
\hline Age (mth), mean (SD) & $22.5(15.6)$ & $17.3(9.7)$ \\
Weight on admission (kg), mean (SD) & $5.8(1.6)$ & $5.7(1.7)$ \\
Weight for age, no. (\%) & $1(3)$ & $2(6)$ \\
$60-69 \%$ & $31(97)$ & $30(94)$ \\
$<60 \%$ & $22(8.0)$ & $21.3(8.2)$ \\
Duration of diarrhoea before admission & & \\
$\quad$ days), mean (SD) & $13(4)$ & $15(3)$ \\
Stool frequency/day, mean (SD) & $9(28)$ & $8(25)$ \\
Vomiting, no. (\%) & & $32(100)$ \\
Degree of dehydration & $32(100)$ & $130.0(3.3)$ \\
"Some” dehydration, no. (\%) & $129.7(3.1)$ & $3.1(0.3)$ \\
Serum sodium (mmol/1), mean (SD) & $3.1(0.3)$ & $6.1(2.2)$ \\
Serum potassium (mmol/1), mean (SD) & $6.3(2.1)$ & $7(22)$ \\
\% weight loss, mean (SD) & & $8(25)$ \\
Enteropathogens, no. (\%) & $8(25)$ & $2(6)$ \\
Enteropathogenic E coli & $7(22)$ & $2(6)$ \\
Rotavirus & $3(9)$ & $1(3)$ \\
Vibrio cholerae & $2(6)$ & $2(6)$ \\
Shigella flexneri & $2(6)$ & $1(3)$ \\
Salmonella typhimurium & $2(6)$ & $1(3)$ \\
Giardia lamblia & $1(3)$ & $5(16)$ \\
Aeromonus sp. & $1(3)$ & $3(9)$ \\
Klebsiella & $4(13)$ & \\
Mixed pathogens & $2(6)$ & \\
No pathogens & & \\
\hline
\end{tabular}

Table 2 Outcome variables

\begin{tabular}{|c|c|c|c|}
\hline Parameters & $\begin{array}{l}\text { Standard ORS } \\
(n=32)\end{array}$ & $\begin{array}{l}\text { Hypo-osmolar ORS } \\
(n=32)\end{array}$ & $p$ value \\
\hline $\begin{array}{l}\text { No. (\%) patients recovered within } 5 \\
\text { days }\end{array}$ & $29(91)$ & $32(100)$ & $>0.05$ \\
\hline Median survival time to recovery (h) & 53 & 36 & 0.001 \\
\hline $\begin{array}{l}\text { Duration of diarrhoea after initiation } \\
\text { of therapy }(\mathrm{h})\end{array}$ & $66.4(32.3)$ & $41.5(25.1)$ & 0.001 \\
\hline \multicolumn{4}{|l|}{ Stool output } \\
\hline $0-24 \mathrm{~h}(\mathrm{~g} / \mathrm{kg})$ & $105.9(44.6)$ & $73.4(23.1)$ & 0.001 \\
\hline $24-48 \mathrm{~h}(\mathrm{~g} / \mathrm{kg})$ & $87.5(66.5)$ & $34.9(13.5)$ & 0.001 \\
\hline $48-72 \mathrm{~h}(\mathrm{~g} / \mathrm{kg})$ & $90.4(67.7)$ & $28.4(18.0)$ & 0.01 \\
\hline At recovery (g/kg/day) & $96.6(42.8)$ & $52.3(21.3)$ & 0.0001 \\
\hline \multicolumn{4}{|l|}{ ORS intake } \\
\hline $0-24 \mathrm{~h}(\mathrm{ml} / \mathrm{kg})$ & $184.5(53.7)$ & $109.7(32.2)$ & 0.0001 \\
\hline $24-48 \mathrm{~h}(\mathrm{ml} / \mathrm{kg})$ & $151.2(81.3)$ & $73.4(22.7)$ & 0.0001 \\
\hline $48-72 \mathrm{~h}(\mathrm{ml} / \mathrm{kg})$ & $151.5(65.0)$ & $54.9(28.3)$ & 0.001 \\
\hline At recovery $(\mathrm{ml} / \mathrm{kg} /$ day $)$ & $168.9(52.4)$ & $111.5(39.4)$ & 0.0001 \\
\hline $\begin{array}{l}\text { Fluid intake (ORS + water + liquid } \\
\text { food) }(\mathrm{ml} / \mathrm{kg} / \text { day })\end{array}$ & $278.3(99.3)$ & $214.6(61.2)$ & 0.003 \\
\hline $\begin{array}{l}\% \text { of weight gain (\% of admission } \\
\text { weight) }\end{array}$ & $5.4(1.3)$ & $4.3(1.2)$ & 0.001 \\
\hline
\end{tabular}

Results expressed as mean (SD) unless otherwise indicated.

After selecting the children, written consent was obtained from the parents. A complete history was taken from parents; a thorough physical examination was done and they were assessed for dehydration. ${ }^{4}$ The children were weighed unclothed on a balance of $10 \mathrm{~g}$ precision. Nutritional status was assessed by allocating the admission weight (after adjustment for loss caused by dehydration) of the children to different weight for age nutritional groups according to the classification of Indian Academy of Pediatrics. ${ }^{15}$ Stool samples were collected from all children. Bacteriological examination of stool samples and characterisation of the different isolates were carried out using standard techniques. ${ }^{16}$ Microscopic examination of stool samples was performed to detect trophozoites and cysts of Entamoeba histolytica and Giardia lamblia. Stool samples were stored at $-20^{\circ} \mathrm{C}$ and subsequently analysed for rotavirus using enzyme linked immunosorbent assay (ELISA) and polyacrylamide gel electrophoresis (PAGE). Blood samples were collected for estimation of serum sodium and potassium.

Group 1 received hypo-osmolar ORS (sodium $60 \mathrm{mmol} / 1$, potassium $20 \mathrm{mmol} / 1$, chlo- ride $50 \mathrm{mmol} / \mathrm{l}$, glucose $84 \mathrm{mmol} / \mathrm{l}$, citrate 10 $\mathrm{mmol} / \mathrm{l}$ by dissolving sodium chloride $1.75 \mathrm{~g}$, potassium chloride $1.5 \mathrm{~g}$, trisodium citrate dihydrate $2.9 \mathrm{~g}$, and glucose $15 \mathrm{~g}$ in 1 litre of water, osmolarity 224). Group 2 received the standard ORS recommended by WHO/ UNICEF (sodium $90 \mathrm{mmol} / \mathrm{l}$, potassium 20 $\mathrm{mmol} / \mathrm{l}$, chloride $80 \mathrm{mmol} / \mathrm{l}$, glucose 111 $\mathrm{mmol} / \mathrm{l}$, citrate $10 \mathrm{mmol} / \mathrm{l}$ by dissolving sodium chloride $3.5 \mathrm{~g}$, potassium chloride $1.5 \mathrm{~g}$, trisodium citrate dihydrate $2.9 \mathrm{~g}$, and glucose $20 \mathrm{~g}$ in 1 litre of water, osmolarity 311). A computer generated randomisation table was used to allocate the different ORS packets. The table was held by an individual who was not associated with the study and he provided the ORS packets according to the table. The packets of hypo-osmolar ORS and standard ORS were similar in appearance and packaged in identical sachets. Ten 1 litre packets were provided for each child. All children were rehydrated orally within four to six hours using the assigned ORS solution. It was then given to replace continuing losses (liquid stool and vomitus) until diarrhoea stopped (two formed stools passed, or no stool for 12 hours), or for up to five days if diarrhoea persisted. The children were allowed to drink water ad libitum. Plain water was also offered. Breast fed children were allowed to continue breast feeding. Formula milk and animal milk were permitted. Older children received the normal diet which they were used to before this illness. They were not given any drug therapy during the study.

Intake and output were measured and recorded eight hourly until the diarrhoea stopped or for up to five days if it persisted, or until the child was withdrawn from the study. Stool losses were measured on preweighed disposable diapers; urine was separated from stool using urine collection bags. Vomitus losses were evaluated by weighing on preweighed gauze pads. Children were weighed after correction of initial dehydration and every morning between 10.00 and $10.30 \mathrm{am}$. Follow up records were kept on a predesigned proforma. Daily records were kept as follows: (1) number of stools per 24 hours; (2) number of episodes of vomiting; (3) stool weight; (4) vomitus weight; (5) intake of water and other liquid food. Measurement units were sensitive to $1 \mathrm{~g}$ or $1 \mathrm{ml}$. Blood samples were drawn again for estimation of serum sodium and potassium on recovery or on day 5 of hospitalisation if the child did not recover. Children were classified as hypernatraemic if serum sodium was greater than $150 \mathrm{mmol} / 1$ and hyponatraemic if it was less than $130 \mathrm{mmol} / \mathrm{l}$. They were classified as hyperkalaemic and hypokalaemic if serum potassium was greater than 5 or less than $3.5 \mathrm{mmol} / 1$ respectively. Children, other than those who were very ill, were discharged on recovery and the parents were advised to attend the hospital nutrition clinic for nutritional rehabilitation.

After decoding, the two groups were compared using the $\chi^{2}$ test. The means of the outcome variables of the two groups (time specific stool output, intake of ORS, total fluid intake (ORS + water + liquid food), weight gain or loss, and electrolyte concentrations on recov- 
Table 3 Mean serum sodium and potassium concentrations (mmol/l)

\begin{tabular}{|c|c|c|c|c|}
\hline & \multicolumn{2}{|c|}{ Standard ORS $(n=32)$} & \multicolumn{2}{|c|}{ Hypo-osmolar ORS $(n=32)$} \\
\hline & On admission & On recovery & On admission & On recovery \\
\hline Sodium & $129.7(3.1)$ & $134.4(3.1)$ & $130.0(3.3)$ & $134.4(3.1)$ \\
\hline Potassium & $3.1(0.3)$ & $3.5(0.3)$ & $3.1(0.3)$ & $3.5(0.3)$ \\
\hline
\end{tabular}

Results expressed as mean (SD).

There was no difference on admission and on recovery between the groups.

Increases in sodium and potassium in the two groups are the same.

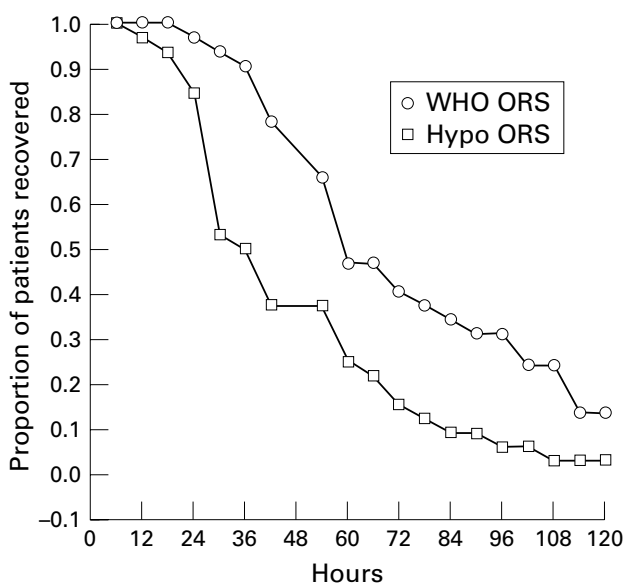

Figure 1 Survival curve for recovery.

ery) were compared by applying Student's $t$ test. The difference in proportions of cured patients between the two groups was examined using the $\chi^{2}$ test. Recovery time of patients in the two groups was calculated using a survival analysis technique in accordance with the Kaplan-Meyer method.

\section{Results}

A total of 64 marasmic male children (aged 6-48 months) suffering from dehydrating acute watery diarrhoea were enrolled in the study. After decoding the identity of ORS received by the two groups, it was observed that 32 children were in the standard ORS and 32 in the hypo-osmolar ORS group. Thirty one children $(97 \%)$ in the standard and $30(94 \%)$ in the hypo-osmolar ORS group had less than $60 \%$ Harvard standard weight for age. One child in the standard and two children in the hypo-osmolar group had 60-69\% Harvard standard weight for age. Table 1 presents clinical features on admission, diarrhoeal pathogens isolated, serum sodium and potassium concentrations, and percentage of weight loss; the groups were comparable. In table 2, outcome variables of the two groups are compared.

Twenty nine children $(91 \%)$ in the standard ORS group and $32(100 \%)$ in the hypoosmolar ORS group recovered within five days; this difference was not statistically significant ( $p>0.05)$. The interval to recovery, stool output, and daily ORS and fluid intake per kg were significantly less in the hypo-osmolar ORS group than in the standard ORS group. Mean percentage weight gain in children in the hypoosmolar ORS group was significantly lower than in the standard ORS group at discharge or on day 5 if they did not recover during this period $(p=0.001)$. Figure 1 shows the survival curve for recovery time in the two groups.
Recovery time of the hypo-osmolar group was significantly less than that of the standard ORS group. Mean serum sodium and potassium concentrations at time of recovery or on day 5 for those who did not recover, were similar in both treatment groups (table 3 ).

\section{Discussion}

This study was designed to compare the clinical efficacy of hypo-osmolar ORS and standard ORS in severely malnourished (marasmic) children with dehydrating acute diarrhoea. It confirms the superiority of hypo-osmolar ORS over standard ORS. It has shown for the first time that hypo-osmolar ORS is safe, and more effective than standard ORS in marasmic children with "some" dehydration. Hypo-osmolar ORS significantly decreases the mean duration of diarrhoea, stool output, and need for ORS and other fluids during the course of treatment compared to that of standard ORS. The beneficial effect of hypo-osmolar ORS may be a result of low osmolality of the solution and complete absorption of glucose, thus reducing the risk of osmotic diarrhoea.

The results showed that rehydration could be achieved and hydration status maintained with hypo-osmolar ORS as effectively as standard ORS. None of the children in either group became over-hydrated in the course of treatment. Several studies of hypo-osmolar ORS in acute diarrhoea have documented reduced weight gain in children on hypo-osmolar compared to standard ORS, but this was not statistically significant. ${ }^{10-13}$ In contrast, our study showed that the mean percentage weight gain in children in the hypo-osmolar ORS group was significantly lower $(p=0.001)$ compared to the standard ORS group on recovery, reflecting the lower consumption of hypoosmolar ORS for correction of dehydration as well as for maintenance.

Others have cautioned against the use of standard ORS because of the potential risk of hypernatraemia. ${ }^{17-21} \mathrm{~A}$ study conducted in Calcutta showed that standard ORS could be used safely and effectively for the treatment of dehydrating diarrhoea in marasmic children with the provision of an additional source of free water (plain water, breast milk, or other low solute feeds). ${ }^{6}$ WHO experts have also recommended the use of standard ORS together with additional fluid in the form of breast milk, dilute milk formula, or plain water in the latter part of rehydration, as well as during the maintenance phase to reduce the risk of hypernatraemia. ${ }^{4}$ However, if hypo-osmolar ORS is available, it can be used safely and easily for the treatment of marasmic children.

In developing countries, it has been thought that use of hypo-osmolar ORS in marasmic diarrhoeal children might cause hyponatraemia, because these children are already sodium depleted, and furthermore acute diarrhoea may be caused by various bacterial pathogens which may induce high stool sodium losses. However, the present study shows that hyponatraemia was present at the time of admission in $15(47 \%)$ children in the hypo-osmolar group, but it was corrected using the low 
sodium solution. This can be explained by the fact that although serum sodium may be low in marasmic children, total body sodium may be normal.

We conclude that hypo-osmolar ORS is superior to standard ORS for the treatment of dehydrating acute watery diarrhoea in severely malnourished (marasmic) children. Hypoosmolar ORS resulted in a shorter duration of diarrhoea, a reduced stool output, less need for maintenance therapy, and a reduced chance of hypernatraemia. On the basis of this study on malnourished children and previous studies in well-nourished children, a general recommendation is made that hypo-osmolar ORS (sodium content $60 \mathrm{mmol} / \mathrm{l}$ ) should be used for rapid rehydration, and maintenance of hydration in children with non-cholera diarrhoea.

We acknowledge Drs Bipul Chandra Roy, Soumyadip Das Gupta and Shanta Dutta for their help in clinical and microbiologica work; Mr Mahendra Mullick and Milan Dey for technical assistance; and Mr Shyamal Kumar Das for secretarial help.

1 Chen LC, Rahman M, Sardar AM. Epidemiology and causes of death among children in rural area of Bangladesh. Int $\mathcal{F}$ Epidemiol 1980;9:25-33.

2 Kamath KR, Feldman RA, Rao PSSS, Webb JKG. Infection and disease in a group of South Indian families. II. General morbidity patterns in families and family members. $A m \mathcal{F}$ Epidemiol 1969;89:375-83.

3 James JW. Longitudinal study of the morbidity of diarrhoeal and respiratory infections in malnourished children. $A m \mathcal{F}$ Clin Nutr 1972;25:690-4.

4 World Health Organization. Programme for the control of diarrhoeal diseases: a manual for the treatment of diarrhoea. $\mathrm{WHO}$ CDD/SER/80.2 Rev 2. Geneva: WHO, 1990.

5 Chatterjee A, Mahalanabis D, Jalan KN, et al. Oral rehydration in infantile diarrhoea: control trial of a low sodium glucose electrolyte solution. Arch Dis Child 1978;53:284-9.
6 Dutta P, Bhattacharya SK, Dutta D, et al. Oral rehydration solution containing 90 millimol sodium is safe and useful in treating diarrhoea in severely malnourished children. $\mathcal{F}$ Diarrhoeal Dis Res 1991;9:118-22.

7 Klahr S, Alleyne AOG. Effects of chronic protein calorie malnutrition on the kidney. Kidney Int 1973;3:129-41.

8 Nichols BL, Alvarado MJ, Radrigua SJ, et al. Therapeutic implications of electrolyte, water and nitrogen losses during recovery from protein-calorie malnutrition. 7 Pediatr 1974; 84:759-68.

9 Alley GAO. The effect of severe protein calorie malnutrition on renal function of Jamaican children. Pediatrics 1967;39: 400-11.

10 Rautanen T, El-Radhi S, Vesikari T. Clinical experience with a hypotonic oral rehydration solution in acute diarrhoea. Acta Paediatr 1993;82:52-4.

11 International study group on reduced-osmolarity ORS solutions. Multicentre evaluation of reduced-osmolarity oral rehydration salts solution. Lancet 1995;345:282-5.

12 Mahalanabis D, Faruque ASG, Hoque SS, Faruque SM. Hypotonic solution in acute diarrhoea: a controlled clinical trial. Acta Pediatr 1995;84:289-93.

13 Rautanen T, Salo E, Verkasalo M, Vesikari T. Randomised double blind trial of hypotonic oral rehydration solutions with or without citrate. Arch Dis Child 1994;70:44-6.

14 El-Mougi M, El Akkad W, Hendawi A, et al. Is a low osmolarity ORS solution more efficacious than standard WHO ORS Solution? F Pediatr Gastroenterol Nutr 1994;19:83-6.

15 Nutrition sub-committee of Indian Academy of Pediatrics. Report of the Convener. Indian $\mathcal{F}$ Pediatr 1972;9:360.

16 World Health Organization. Manual for laboratory investigation of acute enteric infection. Programme for control of diarrhoeal diseases. CDD/83.3. Geneva: WHO, 1983.

17 Finberg L, Harper HA, Harrison HE, Black RE. Oral rehydration for diarrhoea. F Pediatr 1982;101:497-9.

18 Nichols BL, Soriano HA. A critique of oral therapy of dehydration due to diarrhoeal syndrome. Am f Clin Nutr 1977; 30:1457-72.

19 Hirschhorn N. The treatment of acute diarrhoea in children: a historical and physiological perspective. $A m \mathcal{F}$ Clin Nutr 1980;33:637-63.

20 Samadi AR, Wahed MA, Islam MR, Ahmed SM. Consequences of hyponatraemia and hypernatraemia in children with acute diarrhoea in Bangladesh. BMF 1983;286:671-3.

21 Cleary TG, Cleary KR, DuPont HL, et al. Relationship of oral rehydration solution to hypernatraemia in infantile diarrhoea. F Pediatr 1981;99:739-41. 